\title{
Spectrophotometry for Xanthochromia
}

TO THE EDITOR: Xanthochromia, or a yellow appearance of the cerebrospinal fluid, is often used to confirm suspected subarachnoid hemorrhage. ${ }^{1,2}$ It is associated with the presence of bilirubin, a blood product that arises only in vivo by the enzymatic transformation of hemoglobin. However, visual assessment of the cerebrospinal fluid for xanthochromia is highly subjective and unreliable, especially in cases in which other blood products, such as oxyhemoglobin, are present. ${ }^{1,2}$ The differential diagnosis may be further complicated by a high protein level and a high cell count, as in meningitis and necrosis, or by the presence of other pigments (namely, carotenoids). To distinguish pigments in the cerebrospinal fluid, such as bilirubin, that are clinically important for diagnosing subarachnoid hemorrhage and other types of intracerebral bleeding from

Figure 1. Chromaticity Diagram.

A section of the Commission Internationale de l'Eclairage (CIE) 1931 chromaticity diagram is shown, with the $x-y$ chromaticity coordinates of 72 samples of cerebrospinal fluid containing bilirubin (yellow dots) with respect to CIE illuminant D65 (white cross). For reference, the $x-y$ chromaticity coordinates and the wavelengths of the spectral colors from $550 \mathrm{~nm}$ (corresponding to greenish yellow) to $620 \mathrm{~nm}$ (corresponding to red) are indicated. The region corresponding to pure yellow, which ranges between 575 and $580 \mathrm{~nm}$ (as noted by Petzold and Sharpe ${ }^{4}$ ), is shown in gray. The pie chart indicates the proportions of samples visually identified as greenish, yellow (xanthochromic), and reddish by trained clinical observers. The full chromaticity diagram is shown in the lower left-hand corner. those that are not important, objective spectrophotometry, rather than visual assessment, is recommended. Because bilirubin has a characteristic signature - absorbance in the blue range (450 to 460 $\mathrm{nm})$ - it can be readily identified by spectrophotometry. Moreover, by standard colorimetric procedures, which have been established by the Commission Internationale de l'Eclairage (CIE, or the International Commission on Illumination), the

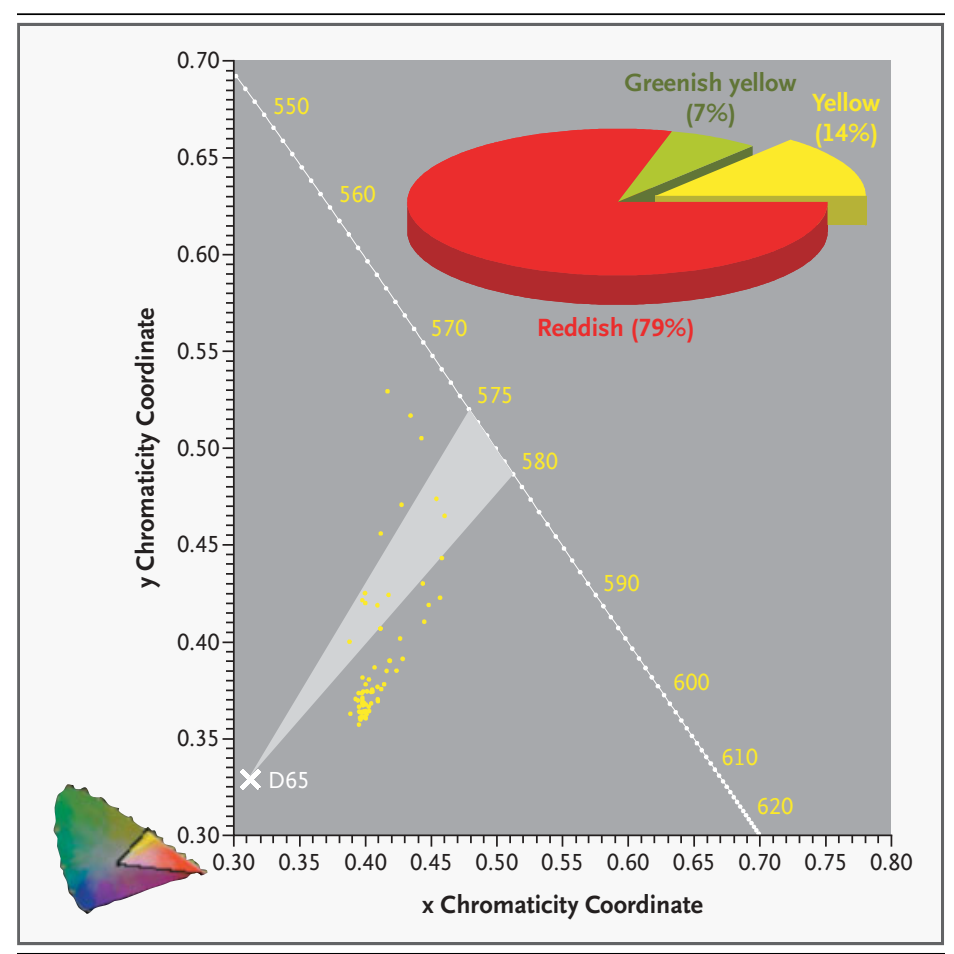


spectrophotometric trace can be converted to its chromaticity coordinates. ${ }^{3}$ These procedures allow the trace to be plotted in a geometric diagram (the CIE 1931 chromaticity diagram for the 2-degree field of view) and allow its dominant wavelength (corresponding to hue) to be defined with respect to average daylight conditions (CIE standard illuminant D65) (Fig. 1).

Spectrophotometric analysis of 632 samples of cerebrospinal fluid, obtained at the National Hospital for Neurology and Neurosurgery in London between January 1996 and April 2004, indicated that 72 contained bilirubin. Of these, only 15 (21 percent) contained bilirubin alone and appeared pure yellow (14 percent) or greenish yellow (7 percent) (Fig. 1). A significantly higher number, 57 (79 percent; chi-square $=49.0, \mathrm{P}<0.001$ ), contained oxyhemoglobin as well as bilirubin. Their appearance ranged from red and reddish pink to orange. These findings reveal that about 80 percent of cerebrospinal fluid samples containing substantial amounts of bilirubin are not typically perceived as xanthochromic (i.e., yellow). Equally important, the presence of bilirubin cannot reliably be ruled out in the case of cerebrospinal fluid samples that are visibly red.

In the United Kingdom, the rate of visual assessment of the cerebrospinal fluid fell from 24 percent to 6 percent, whereas the use of spectrophotometry rose from 76 percent to 94 percent, after the introduction of new guidelines for the detection of cerebrospinal fluid pigments. This shift has reduced the estimated error rate from 40 percent to 9 percent. ${ }^{5}$
Clearly, xanthochromia on the basis of mere visual assessment is an ambiguous concept clinically, physiologically, and biochemically. Two recent surveys, however, have shown that visual assessment of cerebrospinal fluid samples for xanthochromia is still the most common method used in the United States. ${ }^{2,6}$ We believe that this practice should immediately be reevaluated.

Axel Petzold, M.D., Ph.D.

National Hospital for Neurology and Neurosurgery London WCIN 3BG, United Kingdom

a.petzold@ion.ucl.ac.uk

Geoffrey Keir, Ph.D.

Institute of Neurology

London WCIN 3BG, United Kingdom

Lindsay T. Sharpe, Ph.D.

Institute of Ophthalmology

London ECIV 9EL, United Kingdom

1. UK National External Quality Assessment Scheme for Immunohistochemistry Working Group. National guidelines for analysis of cerebrospinal fluid for bilirubin in suspected subarachnoid haemorrhage. Ann Clin Biochem 2003;40:481-8.

2. Edlow JA, Bruner KS, Horowitz GL. Xanthochromia. Arch Pathol Lab Med 2002;126:413-5.

3. Wyszecki G, Stiles WS. Color science: concepts and methods, quantitative data and formulae. 2nd ed. New York: John Wiley, 1982. 4. Petzold A, Sharpe LT. Hue memory and discrimination in young children. Vision Res 1998;38:3759-72.

5. Patel $D$, White $P$, Egner $W$, Beetham R. National guidelines for analysis of CSF bilirubin and their impact. In: Proceedings of the Association of Clinical Biochemists National Meeting, Birmingham, England, May 18-20, 2004. abstract.

6. Judge B. Laboratory analysis of xanthochromia in patients with suspected subarachnoidal hemorrhage: a national survey. In: Proceedings of the American College of Emergency Physicians Scientific Assembly, Philadelphia, October 23-25, 2000. abstract.

Correspondence Copyright $\odot 2004$ Massachusetts Medical Society.

\section{INSTRUCTIONS FOR LETTERS TO THE EDITOR}

Letters to the Editor are considered for publication, subject to editing and abridgment, provided they do not contain material that has been submitted or published elsewhere. Please note the following: $\cdot$ Letters in reference to a Journal article must not exceed 175 words (excluding references), must be received within three weeks after publication of the article, and must be submitted over the Internet at http://authors.nejm.org. Letters not related to a Journal article must not exceed 400 words and may be submitted over the Internet or sent, typewritten and triple-spaced, by mail. $\cdot A$ letter can have no more than five references and one figure or table. $\cdot$ A letter can be signed by no more than three authors. $\cdot$ Financial associations or other possible conflicts of interest must be disclosed. (Such disclosures will be published with the letters. For authors of Journal articles who are responding to letters, this information appears in the original articles.) . Include your full mailing address, telephone number, fax number, and e-mail address with your letter.

Our address: Letters to the Editor $\cdot$ New England Journal of Medicine $\cdot 10$ Shattuck St. $・$ Boston, MA 02115

Our Web address: http://authors.nejm.org

Our fax numbers: 617-739-9864 and 617-734-4457

We cannot acknowledge receipt of your letter, but we will notify you when we have made a decision about publication. Letters that do not adhere to these instructions will not be considered. Rejected letters and figures will not be returned. We are unable to provide prepublication proofs. Submission of a letter constitutes permission for the Massachusetts Medical Society, its licensees, and its assignees to use it in the Journal's various print and electronic publications and in collections, revisions, and any other form or medium. 